\title{
Machine Interpretation of 2D Line Drawings as 3D Solid Objects
}

\author{
P. A. C. Varley \\ Department of Precision Engineering, The University of Tokyo, Tokyo, Japan
}

\begin{abstract}
In engineering design, much time and effort could be saved if a computer could interpret an engineer's initial concept drawings.

This paper describes a prototype system for interpreting line drawings as solid polyhedral objects. It concentrates on four stages of processing: line labelling, identification of parallel lines, inflation to $2 \frac{1}{2} D$, and determination of hidden topology.

Although the system is often successful, particularly with simple drawings, there are also cases where the correct interpretation is obvious to a human but cannot be determined using existing algorithms. The paper gives examples both of the successes of the approach and of typical cases where it cannot replicate human skill.
\end{abstract}

\section{Keywords}

Line Drawing Interpretation, Engineering Design

\section{Introduction}

This paper outlines a method of interpreting 2D line drawings as $3 \mathrm{D}$ solid models. More details of the method, including the topics not covered in this paper, and a discussion of alternative approaches can be found in [19].

Studies such as Jenkins [7] have shown that it is common practice for design engineers to sketch their ideas on paper before entering them into a CAD package. Clearly, valuable time and effort could be saved if a computer could interpret the engineer's initial concept drawings as solid models. Furthermore, if this conversion could be done within a second or two, it would give helpful feedback, further enhancing the designer's creativity [4].

The problem is to produce the a model of the 3D object the engineer would regard as the most reasonable interpretation of the 2D drawing, and to do so quickly. Obviously, there are infinitely many objects

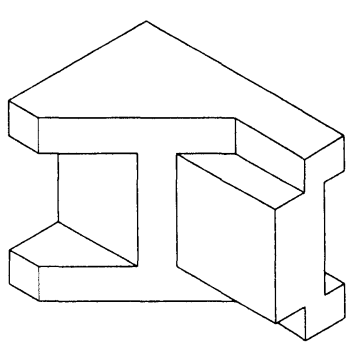

Figure 1. Trihedral Drawing [23]

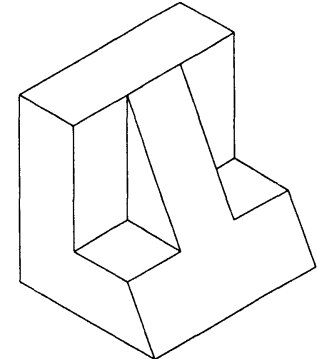

Figure 2. NonTrihedral Drawing [23] which could, if viewed from a particular viewpoint, result in drawings such as Figures 1 and 2. In practice, an engineer would be in little doubt as to which was the correct interpretation.

For this reason, the problem is as much heuristic as geometric: it is not merely to find a geometricallyrealisable solid which corresponds to the drawing, but to find the one which corresponds to the engineer's expectations.

The following approach has been successful in several cases:

- Label the lines in the drawing, to determine which are convex, which are concave, and which are occluding. This is described in Section 2.

- Determine which pairs of lines in the drawing are intended to be parallel in 3D. This is described in Section 3.

- Inflate the drawing to $2 \frac{1}{2}$ by determining $z$ coordinates for each vertex. This is described in Section 4.

- Determine any symmetry elements (mostly planes of reflection) in the object. This is not strictly necessary - the approach described in this paper would in most cases work as well without any symmetry information-and is not described in this 
paper. Determining candidate symmetry elements is straightforward.

- Classify the drawing (e.g. extrusion, normalon, general case). This is straightforward and not described in this paper.

- Complete the object topology by determining the topology of the hidden part of the object. This is described in Section 5.

- Tidy the geometry of the completed object. This is the "beautification of solid models" problem, which has applications outside the field of line drawing interpretation, such as reverse engineering. As such, it is an area of active research in its own right and it is not described in this paper.

Conversion of freehand sketches to line drawings is also beyond the scope of this paper and is not described.

\section{Which Lines are Convex/Concave}

Line labelling is the process of determining whether each line in the drawing represents a convex edge, a concave edge or an occluding edge. For drawings of trihedral objects, ${ }^{1}$ the line labelling problem was essentially solved by Huffman [6] and Clowes [1], who elaborated the catalogue of valid trihedral junction labels. This translates line labelling into a discrete constraint satisfaction problem where the constraints are the 1node constraint that each junction must have a label in the catalogue and the 2-node constraint that each line must have the same label throughout its length.

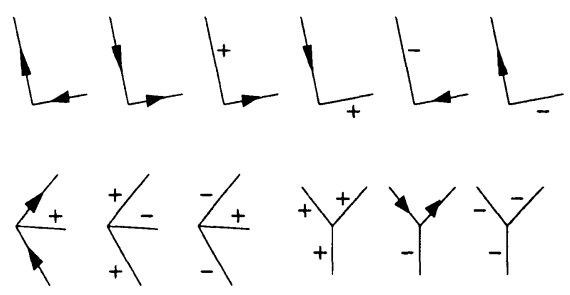

Figure 3. Clowes-Huffman Catalogue

The Clowes-Huffman catalogue for $L-, W$ - and $Y$ Junctions is shown in Figure 3. + indicates a convex edge; - indicates a concave edge; an arrow indicates an occluding edge with the occluding face on the righthand side of the arrow.

In trihedral objects, $T$-junctions (see Figure 4) are always occluding.

\footnotetext{
${ }^{1} \mathrm{~A}$ junction is trihedral if three edges meet at the corresponding vertex. A polyhedral object is trihedral if all its vertices are trihedral.
}

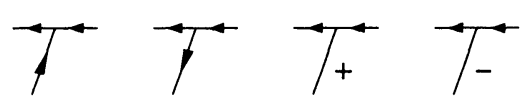

Figure 4. Occluding T-Junctions

For trihedral objects, algorithms for ClowesHuffman line labelling such as those of Waltz [22] or Kanatani [8], although $O\left(e^{n}\right)$ in theory, are usually $O(n)$ in practice [15]. It is believed that the time taken is more a function of the number of legal labellings than of the algorithm, and for trihedral objects there is often only a single legal labelling. For example, Figure 1 has only one valid labelling if the trihedral (ClowesHuffman) catalogue is used.

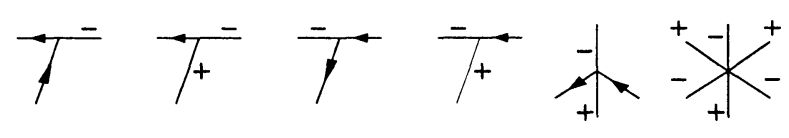

Figure 5. Extended Trihedral Junctions

Extending line labelling algorithms to non-trihedral normalons is easy [14]. The additional legal junction labels are those shown in Figure 5. Note that a new problem has been introduced: the new $T$-junctions are not occluding.

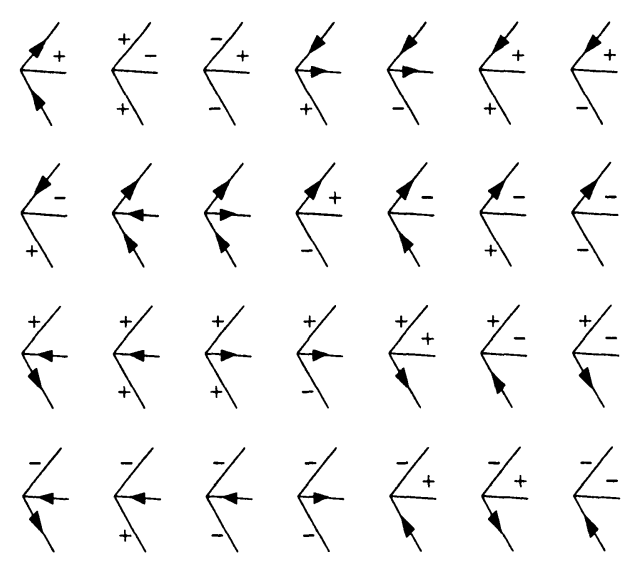

Figure 6. 4-Hedral $W$-Junctions

Extension to the 4-hedral general case is less easy. The catalogue of 4-hedral junction labels is much larger [21] - for example, Figure 6 shows only the possibilities for $W$-junctions.

Because the 4-hedral catalogue is no longer sparse, there may be many valid labellings for a drawing. For example, if the full 4-hedral catalogue is used for all junctions in Figure 2, it has over 200000 valid labellings. Non-trihedral line labelling using the simple combinatorial algorithms used for solving discrete 
CSPs is $O\left(e^{n}\right)$ in practice as well as in theory, and can be very slow. Furthermore, choosing the best of these labellings is not straightforward either, although there are heuristics which can help (see [21]).

An alternative which has been investigated for nontrihedral line labelling is to use a Relaxation algorithm instead. Label probabilities are maintained for each line and each junction, and these probabilities are iteratively updated. If a probability falls to 0 , that label is removed; if a probability reaches 1 , that label is chosen and all competing labels are removed.

In practice, this relaxation algorithm is much faster-labels which are possible but very unlikely are removed quickly by relaxation, whereas they are not removed at all by the combinatorial algorithm. However, relaxation methods are less reliable, since the heuristics for choosing between valid labellings are reasonably effective. Of a test set of 535 line drawings, combinatorial labelling labelled 428 entirely correctly, whereas relaxation labelling only labelled 388 entirely correctly.
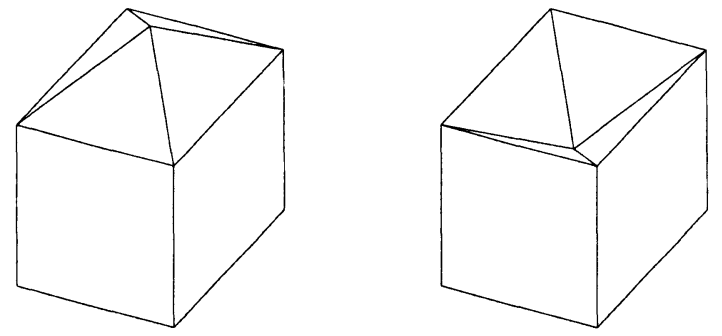

Figure 7. Same Topology

The most serious problem with either method is that in treating line labelling as a CSP, the geometry of the drawing has no effect on the resulting labelling, so (for example) two drawings in Figure 7 will be labelled the same.

Although there are trihedral drawings which can be labelled incorrectly if geometry is ignored, the problems created by ignoring geometry are particularly acute for drawings with several non-trihedral junctions, and for these, other methods are required. Three conference papers describing aspects of the problems of ignoring geometry, and of various proposed solutions making use of the concept outlined here, are currently under review.

\section{Which Lines are Parallel?}

If user inaccuracies are allowed, this is not an easy problem. It is, for example, obvious to a human which lines in Figures 8 and 9 are intended to be parallel in 3D and which are not, but determining this algorithmically presents problems.

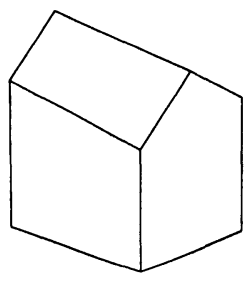

Figure 8.

Badly-drawn House

Some progress has been made. The two drawings can be interpreted correctly by means of two rules:

- Two lines cannot be parallel in 3D if one leaves a face (i.e. has one end in the face and the other not) of which the other line is a part (see Figure 8).

- Two lines cannot be parallel if they leave a face on the same side in 3D but in opposite directions in 2D (see Figure 9) or vice versa. Note that this determination can be made from a successful labelling, and for this reason grouping of parallel lines follows line labelling.

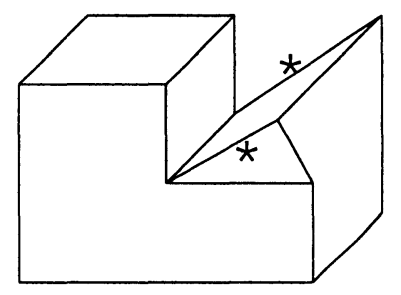

Figure 10. Accidental Coincidence

However, there remain outstanding problems-for example, no existing method can detect that the accidental coincidence in Figure 10 should not result in parallel lines.

Despite such occasional failures, existing methods are good on the whole. However, it is hard to extend them except on a case-by-case basis. For further progress, new ideas are needed. Alternatively, it is possible that parallel lines could be identified as part of the sketch input process, as much potentially useful information is lost in the conversion from freehand sketch to line drawing.

\section{Inflation to $2 \frac{1}{2} \mathrm{D}$}

Inflation is the process of converting a flat $2 \mathrm{D}$ drawing into $2 \frac{1}{2} \mathrm{D}$ by assigning $z$-coordinates (depth coordinates) to each vertex, producing a frontal geometry. 
The approach taken here is the simplest: use compliance functions [11] to generate equations linear in vertex depth coordinates, and solve the resulting linear least squares problem.

Many compliance functions can all be translated into linear equations in $z$-coordinates. Of these, the most useful are:

Perkins's Cubic Corners [16], sometimes called corner orthogonality, assumes that a $W$-junction or $Y$ junction corresponds to a vertex at which three orthogonal faces meet. See Figure 11: the equation relating depth coordinates $z_{V}$ and $z_{A}$ to angles $F$ and $G$ is linear in the $z$-coordinates.
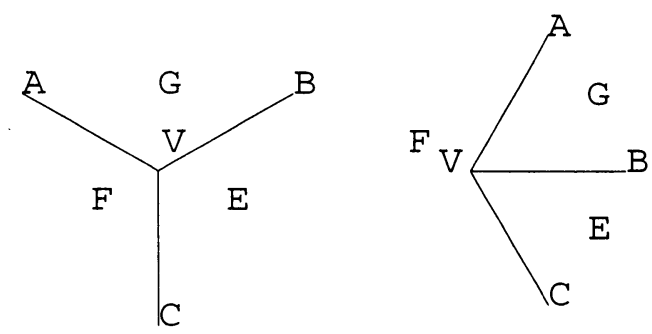

Figure 11. Cubic Corners

Line Parallelism assumes that two edges have been identified as being parallel in $3 \mathrm{D}$. The equation relating the four $z$-coordinates of the vertices at either end of the two edges is obviously linear in the $z$-coordinates. Line parallelism is not, by itself, inflationary: there is a trivial solution ( $z=0$ for all vertices) which meets line parallelism constraints.

Vertex Coplanarity assumes that four vertices have been identified as being coplanar. Again, the equation relating the $z$-coordinates of the four vertices is obviously linear, and the coefficients are easily obtained from 2D geometry. Vertex coplanarity is also not, by itself, inflationary: the trivial solution $(z=0$ for all vertices) also meets vertex coplanarity constraints.

General use of four-vertex coplanarity is not recommended (Lipson [10] points out that where three consecutive vertices on a face are collinear, successive use of four-vertex coplanarity does not guarantee a planar face). However, it is invaluable for cases such as those shown in Figure 12, where without it the linear system of depth equations would be disjoint, with infinitely many solutions.

Lipson and Shpitalni [11] list these and several other compliance functions.

Junction-Label Pairs (an original idea from my $\mathrm{PhD}$ thesis [19]) assume that pairs of junctions with particular labels have the same depth implications they would have in the simplest possible drawing containing this pair. It generates an equation relating the vertex
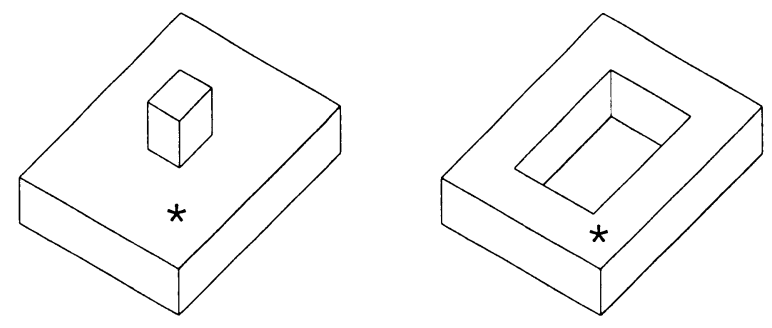

Figure 12. Coplanar Vertices

depths of the ends of the line based on the junction labels of those vertices. For example, see Figure 13: this pair of junction labels can be found in a drawing of a cube, and the implication is that the $Y$-junction is nearer to the viewer than the $W$-junction, with the ratio of $2 \mathrm{D}$ line length to depth change being $\sqrt{2}: 1$.

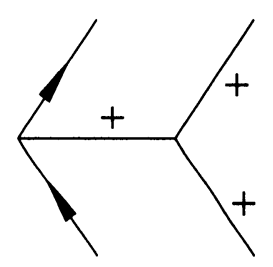
tion Label Pair
Figure 13. Junc-

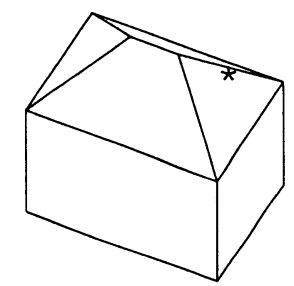

Figure 14. Incorrect Inflation?
Given correct inputs, inflation is the most reliable of the stages of processing described in this paper. Although it occasionally fails to determine correctly which end of a line should be nearer the viewer, such failures are in cases such as in Figure 14 where a human would also have difficulty. The only systematic case where the approach of a linear system of compliance functions fails is that of the Platonic and Archimedean solids, and a known special-case method (Marill's MSDA [13]) is successful for these.

However, two of the most successful compliance functions, line parallelism and junction line pairs, require input information (line parallelism and line labels respectively) which, as already noted, cannot always reliably be obtained.

\section{Determine Hidden Topology}

Once the frontal geometry has been determined, the next stage of processing is to add the remaining topology. The method is essentially that presented in [18]: firstly, add extra edges to complete the wireframe, and then add faces to the wireframe to compete the object. is as follows: 
- While the wireframe is incomplete:

- Project hypothesised edges at each incomplete vertex along the appropriate axes

- Eliminate any edges which would be visible at their points of origin

- Find the locations where the remaining edges intersect, assigning merit figures according to how certain it is that the edges intersects at this location (e.g. an edge which intersects only one other edge gives a higher merit figure than an edge which intersects two or more other edges)

- Reduce the merit for any locations which would be visible (if drawing errors were not allowed, such locations could be eliminated)

- Find the location at which the crossing merit is greatest

- Add a vertex at this location, and the hypothesised edges which crossed at this location, to the known object topology

The process of completing the wireframe topology varies in difficulty depending on the type of object portrayed in the drawing. This paper illustrates two special-case types of object, extrusions and normalons, and the general case. In some cases (e.g. where the object is symmetrical or includes a recognised feature), more than one vertex can be added in one iteration; these are described in [19]. Such cases help both to speed up the process of determining the complete wireframe and to make it more reliable.
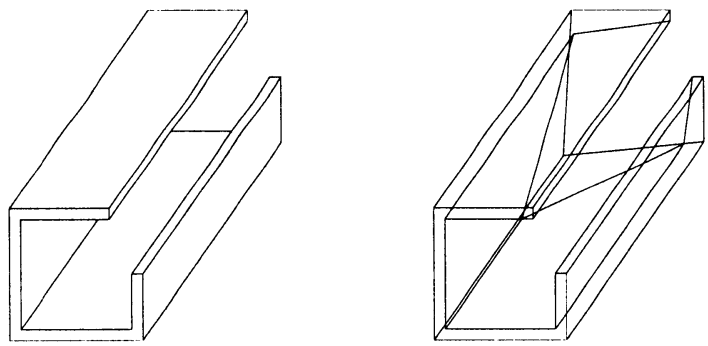

Figure 15. Extrusion

Completing the topology of extrusions from a known front end cap is straightforward. Figure 15 shows a drawing and the corresponding completed extrusion wireframe.

The knowledge that the object is a normalon makes it easier to reconstruct the wireframe correctly, since when hypothesised edges are projected along axes, there will usually be only one edge projected from any
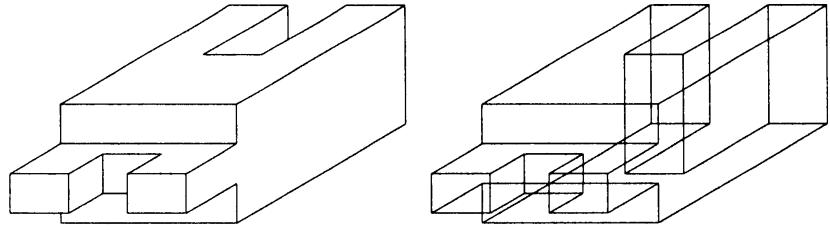

Figure 16. Normalon [23]

particular incomplete vertex. Figure 16 shows a drawing of a normalon and the corresponding completed wireframe.
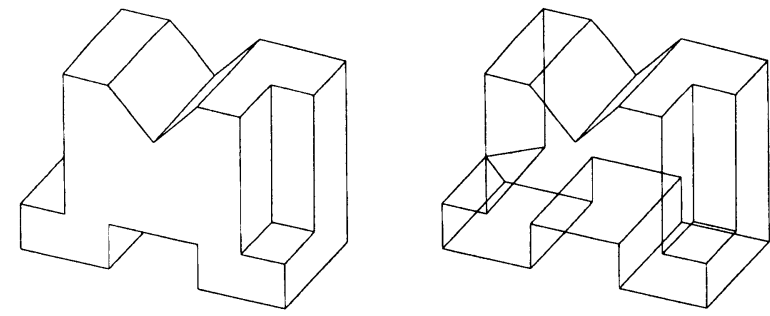

Figure 17. Trihedral Object [23]

Similarly, the knowledge that the object is trihedral helps reduce the number of possible edges projected at each vertex-again, there will be at most one from each incomplete vertex - and thus helps in reconstructing the correct wireframe. Figure 17 shows a drawing of a trihedral object and the corresponding completed wireframe.
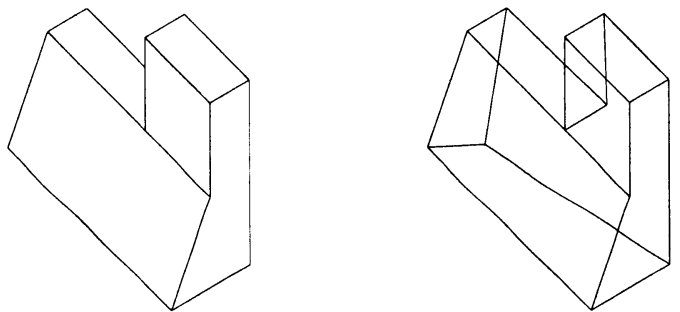

Figure 18. General Case Object

However, in the general case, where the object is neither a normalon nor trihedral, there is the significant difference that hypothesised edges may be projected in any direction parallel to an existing edge. Even after eliminating edges which would be visible, there may be several possibilities at any particular incomplete vertex. In practice, the large number of possible options rapidly becomes too confusing and it is easy to choose an incorrect crossing-point at an early stage. Although such errors can sometimes be rectified by backtracking, the more common result is a valid but unwanted wire- 
frame. Only very simple drawings can be processed reliably. Figure 18 shows a general-case object and the corresponding completed wireframe; this represents the limit of the current state of the art.

Investigations are planned into better (faster and more reliable) ways of determining the correct hidden topology of an object.

Adding additional faces to a wireframe topology for which the frontal geometry is already known is straightforward. I use repeated applications of Dijkstrs Algorithm [2] to find the best loop of unallocated half-edges for each added face, where the merit for a loop of halfedges is based both on the number of half-edges required (the fewer, the better) and their geometry (the closer to coplanar, the better).

\section{Acknowledgements}

I am grateful to the Computer Science Department of Cardiff University for providing the funding for the research described in this paper, and to Japan Society for the Promotion of Science for funding the proposed investigations into improved methods for frontal geometry and construction of topology.

\section{References}

[1] M.B. Clowes, On Seeing Things, Artificial Intelligence, 2, 79-116, 1970.

[2] E.W. Dijkstra, A Note on Two Problems in Connexion with Graphs, Numerische Mathematik I, 269-271, 1959.

[3] S.W.Draper, Reasoning about depth in line-drawing interpretation, $\mathrm{PhD}$ Thesis, Sussex University, 1980.

[4] I.J.Grimstead, Interactive Sketch Input of Boundary Representation Solid Models, PhD Thesis, Cardiff University, 1997.

[5] A.Guzman, Decomposition of a Visual Scene into Three-Dimensional Bodies, AFIPS Proc. Fall Joint Computer Conference, 33, 291-304, 1968.

[6] D.A.Huffman, Impossible Objects as Nonsense Sentences, Machine Intelligence 6, 295-323, New York American Elsevier, 1971.

[7] D.L.Jenkins, The Automatic Interpretation of TwoDimensional Freehand Sketches, $\mathrm{PhD}$ Thesis, University of Wales College of Cardiff, 1992.

[8] K.Kanatani, Group-Theoretical Methods in Image Understanding, Number 20 in Springer Series in Information Sciences, Springer-Verlag, 1990.
[9] D.Lamb and A.Bandopadhay, Interpreting a 3D Object From a Rough 2D Line Drawing. In ed. A.E.Kaufman, Proceedings of the First IEEE Conference on Visualization '90, 59-66, IEEE, 1990.

[10] H.Lipson, Computer Aided 3D Sketching for Conceptual Design, PhD Thesis, Technion-Israel Institute for Technology, Haifa, 1998.

[11] H.Lipson and M.Shpitalni, Optimization-based Reconstruction of a 3D Object from a Single Freehand Line Drawing, Computer-Aided Design 28(8), 651-663, 1996.

[12] J. Malik, Interpreting Line Drawings of Curved Objects, International Journal of Computer Vision 1, 73-103, 1987.

[13] T.Marill, Emulating the Human Interpretation of Line-Drawings as Three-Dimensional Objects, International Journal of Computer Vision 6(2) 147161, 1991.

[14] P.Parodi, R.Lancewicki, A. Vijh and J.K.Tsotsos, Empirically-Derived Estimates of the Complexity of Labeling Line Drawings of Polyhedral Scenes, Artificial Intelligence 105, 47-75, 1998.

[15] P.Parodi and V.Torre, On the Complexity of Labeling Perspective Projections of Polyhedral Scenes, Artificial Intelligence 70, 239-276, 1994.

[16] D.N.Perkins, Cubic Corners, Quarterly Progress Report 89, 207-214, MIT Research Laboratory of Electronics, 1968.

[17] K. Sugihara, Machine Interpretation of Line Drawings, MIT Press, 1986.

[18] P.A.C. Varley, H. Suzuki, J. Mitani and R.R. Martin, Interpretation of Single Sketch Input for Mesh and Solid Models, International Journal of Shape Modelling 6(2), 207-241, 2000.

[19] P.A.C. Varley, Automatic Creation of BoundaryRepresentation Models from Single Line Drawings, PhD Thesis, Cardiff University, 2002.

[20] P.A.C. Varley and R.R. Martin, Estimating Depth from Line Drawings, in ed. K.Lee and N.Patrikalakis, Proc. 7th ACM Symposium on Solid Modeling and Applications, SM02, 180-191, ACM Press, 2002.

[21] P.A.C. Varley and R.R. Martin, Deterministic and Probabilistic Approaches to Labelling Line Drawings of Engineering Objects, International Journal of Shape Modelling 9(1), 79-99, 2003.

[22] D.M.Waltz, Generating Semantic Descriptions from Drawings of Scenes with Shadows, Tech Rept AI-TR-271, M.I.T., Cambridge USA, 1972.

[23] H.W. Yankee, Engineering Graphics, Prindle, Weber and Schmidt, 1985. 